\title{
Molecular surveillance of Dengue Virus (DENV) and its co-infection with Chikungunya Virus (CHIKV) among febrile patients: A comparative study from South Delhi, India
}

\author{
Rohit Sagar \\ Department of Biotechnology, School of Chemical and Life Sciences, Jamia Hamdard, \\ New Delhi-110062, India \& Department of Life Sciences, School of Sciences, IGNOU, \\ New Delhi -110068 , India \\ Siva Raghavendhar \\ Kusuma School of Biological Sciences, IIT, New Delhi -110016, India \\ Kaustuv Nayak \\ ICGEB-Emory Vaccine Center, ICGEB, New Delhi -110067, India \\ Vineet Jain \\ HAH Centenary Hospital, Jamia Hamdard, New Delhi -110062, India \\ Anmol Chandele \\ ICGEB-Emory Vaccine Center, ICGEB, New Delhi -110067, India \\ Ashok Kumar Patel \\ Kusuma School of Biological Sciences, IIT, New Delhi -110016, India \\ Kamal Kumar Gupta \\ Department of Zoology, Deshbandhu College, University of Delhi, New Delhi -110019 , India \\ Murali-Krishna Kaja \\ ICGEB-Emory Vaccine Center, ICGEB, New Delhi 110067, India \& Department of Pediatrics, \\ Emory University School of Medicine, Atlanta, GA - 30322, USA \\ Pratima Ray \\ Department of Biotechnology, School of Chemical and Life Sciences, Jamia Hamdard, New \\ Delhi -110062 , India \\ Neera Kapoor* \\ Department of Life Sciences, School of Sciences, IGNOU, New Delhi -110068, India \\ *Corresponding author. Email: neerakapoor@ignou.ac.in
}

\section{Article Info}

https://doi.org/10.31018/

jans.v13i2.2616

Received: March 12, 2021

Revised: April 28, 2021

Accepted: May 4, 2021

\section{How to Cite}

Sagar, R. et al. (2021). Molecular surveillance of Dengue Virus (DENV) and its co-infection with Chikungunya Virus (CHIKV) among febrile patients: A comparative study from South Delhi, India. Journal of Applied and Natural Science, $13(2), 433$ - 442. https://doi.org/10.31018/jans.v13i2.2616

\begin{abstract}
Dengue and Chikungunya are two major arboviral infections transmitted worldwide by the mosquitoes, Aedes aegypti and Ae. albopictus. India suffers enormously with both Dengue and Chikungunya as they pose a great public health challenge. The present study aims to evaluate the prevalence of Dengue Virus (DENV), Chikungunya Virus (CHIKV) and DENV/CHIKV coinfection (by Reverse-Transcriptase Polymerase Chain Reaction (RT-PCR)/Enzyme Linked Immunosorbent Assay (ELISA), their clinical features, DENV serotypes and CHIKV specific Immunoglobulin G (IgG) within a 7 years gap in the Delhi population. The study sample included clinically suspected febrile patients ( $\leq 7$ days) sera collected during 2017-2018 ( $n=87$ ) and during 2008-2010 $(n=623)$ from Delhi. Captured ELISA was performed for CHIKV IgG screening and nested PCR was done for DENV serotyping. The percentage prevalence for DENV was significantly higher than CHIKV with $41.38 \%(n=87)$ and $16.1 \%(n=87)$ respectively; interestingly, DENV/CHIKV co-infection was detected in 10.34\% ( $n=9 / 87)$ cases during 2017-2018. Similarly, a high DENV prevalence was observed during 2008-2010 with the prevalence rate of $38.3 \%(69 / 180)$, 34.65\% (35/101) and 47.07\% (161/342), respectively. DENV 1 and DENV 3 were dominant serotype during 2008-2010 and 2017-2018 respectively. We have noticed a high prevalence $(36.67 \%, 22 / 60)$ of the CHIKV IgG antibody in the $2017-2018$ samples. Joint pain was more preferential to CHIKV mono-infection and DENV/CHIKV co-infection compared to DENV mono-infection. The present study highlights the need for active surveillance simultaneously for both DENV and CHIKV and to evaluate the role of CHIKV/DENV co-infections in disease severity in the endemic regions.
\end{abstract}

Keywords: CHIKV, DENV, Co-infection, Clinical features, Serotype 
Sagar, R. et al. / J. Appl. \& Nat. Sci. 13(2), 433 - 442 (2021)

\section{INTRODUCTION}

Acute febrile illness is an undifferentiated fever condition which can be observed in adults as well as children of various age groups. The possible cause may be either of dengue, malaria, leptospirosis, chikungunya, japanese encephalitis, typhoid, rickettsia, influenza A (Hammon et al., 1960; Gubler, 1998; Requena-Castro et al., 2017; Singh et al., 2018). The pathogens causing febrile illnesses like dengue fever, chikungunya fever, malaria., are transmitted by arthropod vectors, precisely the mosquitoes. Diseases caused by arboviruses are responsible for the burden on the health-care system worldwide (Singh et al., 2018). Both Dengue Virus (DENV) (Flavivirus, Flaviviridae) and Chikungunya Virus (CHIKV) (Alphavirus, Alphaviridae) account for major public health concerns due to significant morbidity and mortality (especially in the case of dengue). There are 4 serotypes for the dengue virus (DENV 1-4) (Gubler, 1998). Infection with a heterologous serotype may cause more complications like Dengue Haemorrhagic Fever (DHF) which necessitates the identification of DENV serotypes (Hammon et al., 1960; RequenaCastro et al., 2017) so that appropriate epidemiological data can be maintained and the patient could be provided with necessary health care. These viral infections show similar clinical symptoms like high-grade fever, joint pain, rashes, headache, and vomiting (Taraphdar et al., 2012). However, recent have studies reported that multiple joint pain is the characteristic feature of chikungunya virus infection and may persist for several months in the knee, wrist, finger, and ankles (Simon et al., 2007; Burt et al., 2012). Delhi is an endemic region for DENV infection, therefore, its cases are regularly reported every year. Its recent outbreaks have been reported in 2010, 2013, and 2015 (Kumari et al., 2011; Afreen et al., 2014a; Savargaonkar et al., 2018). A total of 9271 and 7136 dengue cases were reported in 2017 and 2018, respectively, from Delhi \{National Vector Borne Disease Control Programme (NVBDCP)\} while 940 and 407 cases of chikungunya fever were reported during 2017 and 2018 from Delhi (NVBDCP). Chikungunya is a re-emerging arboviral disease that occurs intermittently in the region where dengue is endemic. Due to a lack of appropriate diagnostic kits for CHIKV, there is no robust surveillance system for CHIKV screening so the true disease burden always remains unclear. Alternatively, it is a better idea to screen CHIKV specific IgG in acute samples in addition to CHIKV specific IgM. In dengue-endemic areas, the chances of chikungunya infection may be high as both the viruses are transmitted by common mosquito vectors Aedes aegypti and Aedes albopictus (FuruyaKanamori et al., 2016). Human DENV/CHIKV coinfection may occur through the bite of a co-infected mosquito or due to super-infection (Rosso et al., 2016;
Zaidi et al., 2018). Some studies have reported the prevalence of DENV/CHIKV co-infection from Delhi (9.8\% and $10 \%)$ and West Bengal (22.4\%) (Taraphdar et al., 2012; Singh et al., 2012; Afreen et al., 2014a). The same clinical manifestation of DENV and CHIKV patients makes the diagnosis of DENV/CHIKV coinfected patients very difficult clinically and proper management of patients. Therefore, laboratory-based detection of co-infection is very necessary. Due to common vectors, the same clinical symptoms and overlapping geographical distribution of both infections, the clinical practitioners invariably need to know the prevalence rate of DENV/CHIKV co-infection and to differentiate between dengue and chikungunya infection in the initial stage with the help of appropriate early detection kits. This helps in the strategic management of disease severity through optimal therapeutic agents (Subudhi et al., 2018).

The main objectives of the present study are (i) to determine the prevalence of CHIKV, DENV, and DENV/ CHIKV co-infection in the years 2017-2018 from Delhi and comparison of DENV prevalence with the prevalence from Delhi during 2008-2010 (ii) to evaluate clinical features of DENV, CHIKV, and co-infected (DENV \& CHIKV) patients, (iii) determination of DENV serotyping and (iv) to access the immunological status of CHIKV patients.

\section{MATERIALS AND METHODS}

\section{Ethical clearance}

This study was approved by the Institutional Ethical Committee of Jamia Hamdard, New Delhi, and AlIMS, New Delhi. Blood samples were collected from all patients by taking written informed consent.

\section{Patients and clinical samples}

Blood samples were collected from patients having fever $\leq 7$ days duration, attending the Out/In-patient department of Hakeem Abdul Hameed (HAH) Centenary Hospital, Jamia Hamdard, New Delhi from October 2017 to November 2018. This study was approved by the Institutional Ethical Committee of Jamia Hamdard, New Delhi, and AlIMS, New Delhi. The patient's, details including clinical symptoms were recorded after taking consent from the patients or parents/ guardians of children. A total of 87 patients were enrolled which included 42 from the year 2017 and 45 from the year 2018. At the initial stage of recruitment, samples were screened for the dengue day 1 test $(\mathrm{J}$ Mitra and Co.), and for chikungunya advantage $\operatorname{lgM}$ card (J Mitra and Co.) was used. Additionally, samples were subjected to core stringent laboratory diagnoses as outlined in the next section. We had also collected samples from dengue suspected patients from June 2008 to December 2010, from AllMS, New-Delhi. In 


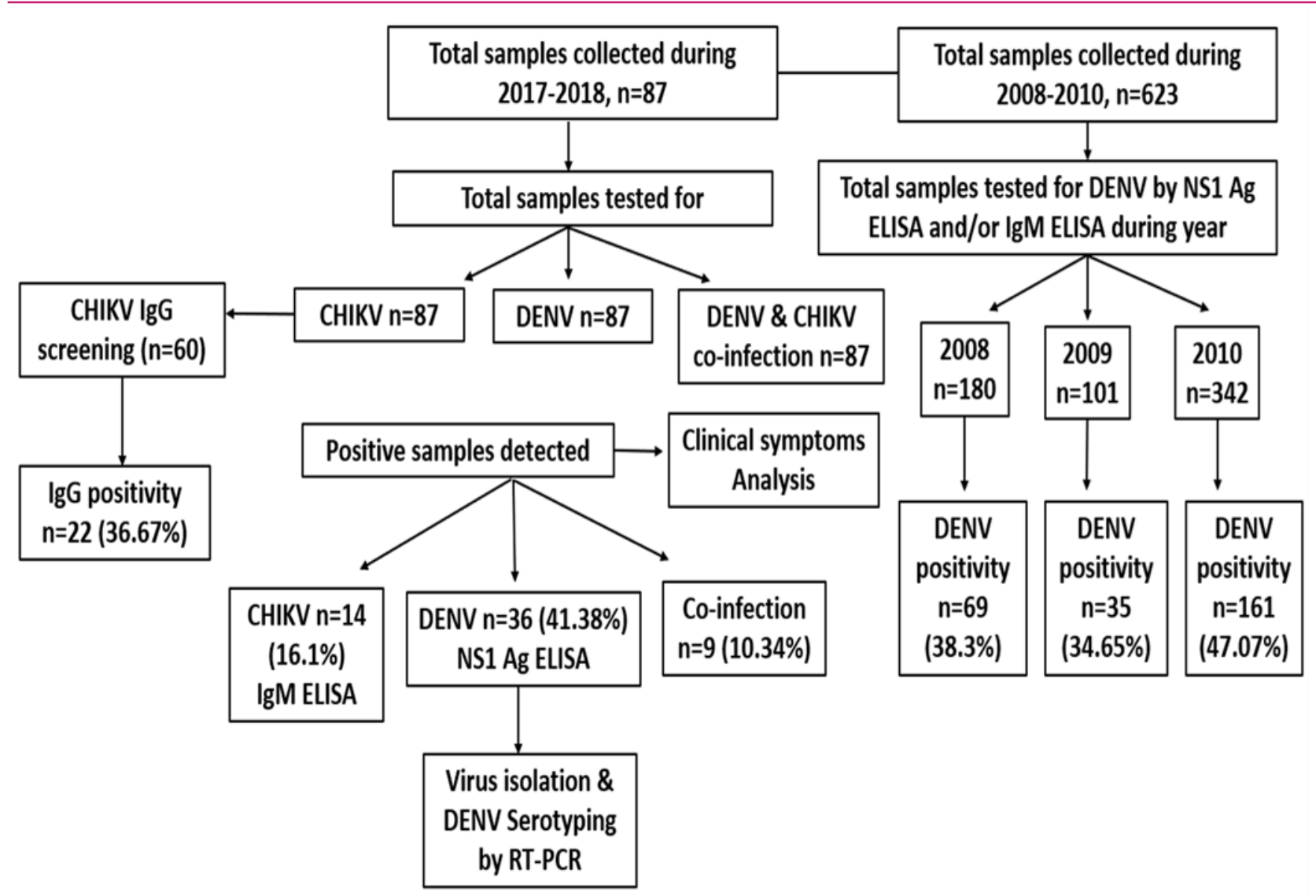

Fig. 1. Schematic representation of patients' plasma samples analyzed for DENV and CHIKV.

2010 Delhi witnessed a dengue fever outbreak so these samples have great importance as far as epidemiology is concerned. A total of 623 acute-phase serum samples were collected having D.O.F $\leq 7$. The total number of collected cases were 180 in 2008, 101 in 2009 , and 342 in 2010 . The study included these samples for comparison of DENV prevalence and its serotyping trend between the years 2017-2018 and 20082010. This is important for a better understanding of DENV epidemiology.

\section{DENV and CHIKV diagnosis}

All the blood samples collected during 2017-2018 were screened for CHIKV infection by using CHIKV RT-PCR and/or an anti-CHIKV IgM ELISA kit (Abcam) (Ray et al., 2012). DENV infection was detected by using the Dengue NS1 Ag ELISA kit by following the manufacturer's protocol (Euroimmun) (Palomares-Reyes et al., 2019) (Fig 1). To assess the CHIKV prior infection, CHIKV specific IgG antibodies were screened by using an anti-CHIKV IgG ELISA kit (Abcam) (Nayak et al., 2020) (Fig 1).

All the blood samples collected during 2008-2010 were tested for dengue virus by IgM ELISA and/or NS1 antigen ELISA (Fig 1). SD BIOLINE Dengue Duo Combo device (Standard Diagnostic Inc., Korea) for IgM ELISA and anti-dengue NS1 based ELISA kit (J. Mitra \& Co.
Pvt. Ltd. India) for NS1 antigen detection were used for DENV screening.

\section{RNA extraction and RT-PCR}

RNA isolation and cDNA synthesis were done by following previously reported protocol (Afreen et al., 2014a). For detection of CHIKV by PCR, published primers were used and the 294 bp E1 gene was amplified and analyzed as described previously (Hasebe et al., 2002).

\section{Determination of DENV serotype}

DENV serotyping was performed for all the NS1 positive samples $(n=36)$ from the $2017-2018$ study period of which 16 samples turned out negative by RT-PCR; therefore, these 16 samples were subjected to virus isolation on the Vero cell line followed by RT-PCR

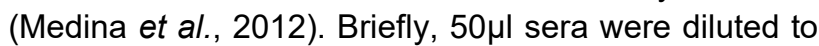
make $200 \mu \mathrm{l}$ volume in plain DMEM media then inoculated in the fresh monolayer of the Vero cell line. Samples were cultured for 3-5 days until the cytopathic effect is observed. After this, the supernatant was collected and carried for RT-PCR cDNA synthesized from NS1 Ag positive samples were subjected to PCR. Firstround PCR was followed by nested PCR. The sizes of the PCR products were $119 \mathrm{bp}$ for DENV 2, $290 \mathrm{bp}$ for DENV 3, and 392 bp for DENV 4 (Lanciotti et al., 1992; 
Table 1. RT-PCR and ELISA based detection of DENV, CHIKV, and DENV/CHIKV co-infection among 87 clinically suspected cases from October 2017 to November 2018 in Delhi.

\begin{tabular}{|c|c|c|c|c|}
\hline \multirow{3}{*}{ Virus } & \multirow{3}{*}{$\begin{array}{l}\text { No. of subjects } \\
\text { tested }\end{array}$} & \multicolumn{3}{|c|}{ No (\%) of positivity } \\
\hline & & ELISA & RT-PCR & Total positivity \\
\hline & & CHIKV IgM /DENV NS1 Ag & CHIKV & \\
\hline $\begin{array}{l}\text { CHIKV } \\
\text { DENV }\end{array}$ & $\begin{array}{l}87 \\
87\end{array}$ & $\begin{array}{l}14(16.1) \\
36^{*}(41.38)\end{array}$ & $\begin{array}{l}0 \\
-\end{array}$ & $\begin{array}{l}14(16.1) \\
36(41.38)\end{array}$ \\
\hline $\begin{array}{l}\text { DENV + CHIKV } \\
\text { co-infection }\end{array}$ & 87 & - & - & $9(10.34)$ \\
\hline
\end{tabular}

Note- Data is no (\%) of patients;

*DENV NS1 positive samples were further taken for serotyping.

Table 2. Comparison of clinical symptoms of CHIKV, DENV mono-infected adults with DENV/CHIKV co-infected adults.

\begin{tabular}{lllllll}
\hline $\begin{array}{l}\text { Clinical } \\
\text { symptoms }\end{array}$ & $\begin{array}{l}\text { CHIKV } \\
\text { mono-infection } \\
(\mathbf{A}=4)\end{array}$ & $\begin{array}{l}\text { DENV } \\
\text { mono-infection } \\
(\mathbf{B}=21)\end{array}$ & $\begin{array}{l}\text { Co-infection } \\
(\mathbf{D E N V}+\mathbf{C H I K V}) \\
(\mathbf{C = 8})\end{array}$ & & \multicolumn{3}{c}{$\boldsymbol{P}$-value } \\
\hline Rash & 0 & $3(14.29)$ & $2(25)$ & 1.000 & 0.515 & 0.597 \\
Headache & $3(75)$ & $19(90.47)$ & $6(75)$ & 0.422 & 1.000 & 0.300 \\
Joint pain & $3(75)$ & $9(42.86)$ & $6(75)$ & 0.322 & 1.000 & 0.215 \\
Joint swelling & $1(25)$ & 0 & $1(12.5)$ & 0.160 & 1.000 & 0.276 \\
Abdominal pain & $1(25)$ & $7(33.33)$ & $2(25)$ & 1.000 & 1.000 & 1.000 \\
Diarrhea & 0 & $3(14.29)$ & $2(25)$ & 1.000 & 0.515 & 0.597 \\
Cough & $1(25)$ & $9(42.86)$ & $3(37.5)$ & 0.627 & 1.000 & 1.000 \\
Vomiting & $2(50)$ & $16(76.19)$ & $4(50)$ & 0.549 & 1.000 & 0.209 \\
Rhinitis & 0 & $2(9.5)$ & 0 & 1.000 & 1.000 & 1.000 \\
\hline
\end{tabular}

Note. Data are no. (\%) of patients; $P$-value was calculated by using the Fischer exact test.

Chahar et al., 2009). Agarose gel electrophoresis (2\%) was used for analyzing the PCR products (Fig 2). Similarly, a total of 69 samples were selected from 20082010 collected samples for DENV serotyping.

\section{Statistical analysis}

Graph Pad Prism software, version 5.2 was used for data analysis. Fischer's exact test was employed to compare the clinical symptoms in CHIKV, DENV, and DENV/CHIKV co-infection. For analysis purposes, we considered the value of $\mathrm{P}<0.05$ as significant (Azeredo et al., 2018).

\section{RESULTS}

\section{Prevalence of CHIKV \& DENV in febrile patients}

A total of 87 sera were tested for chikungunya by both genomic (RT-PCR) and serological based (IgM) test of which $16.1 \%$ were found positive for CHIKV infection only by IgM; none was detected CHIKV RNA positive (Table 1). The median age of CHIKV infected patients was 26 years. Males $(57.14 \%, n=8 / 14)$ were more infected with CHIKV than females $(42.85 \%, 6 / 14)$, although not found statistically significant. In 2017, CHIKV positivity was $9.5 \%$ (4/42), whereas in 2018 , it was $22.22 \%(10 / 45)$. Dengue infection was found in $41.38 \%$ (36/87) cases by NS1 Ag ELISA (Table 1). We did not find any NS1 Ag ELISA positive samples positive by RT -PCR, but we could able to determine DENV serotyping by nested PCR. The median age of DENV infected patients was 24.5 years and males $(55.88 \%, n=19 / 34)$ were more affected than females $(44.12 \%, n=15 / 34)$. DENV infections were $33.33 \%$ (14/42) in the year 2017 and $48.89 \%(22 / 45)$ in the year 2018 while the prevalence of DENV infection was $38.3 \%(69 / 180)$ in 2008 , $34.65 \%(35 / 101)$ in 2009 and $47.07 \%$ (161/342) in 2010 (Fig 3).

\section{Prevalence of anti-CHIKV antibodies in patients during 2017-2018}

Out of total screened samples $(n=87)$, CHIKV specific IgM antibodies were detected in $16.1 \%(14 / 87)$ samples and CHIKV specific IgG antibodies were detected in $36.67 \%(22 / 60)$ samples. This IgG prevalence data gives a rough estimation of previous exposure of patients under study. Only $16.36 \%(9 / 55)$ samples were found positive for both IgM and IgG whereas 9.1\% $(5 / 55)$ samples were detected IgM positive only. The 

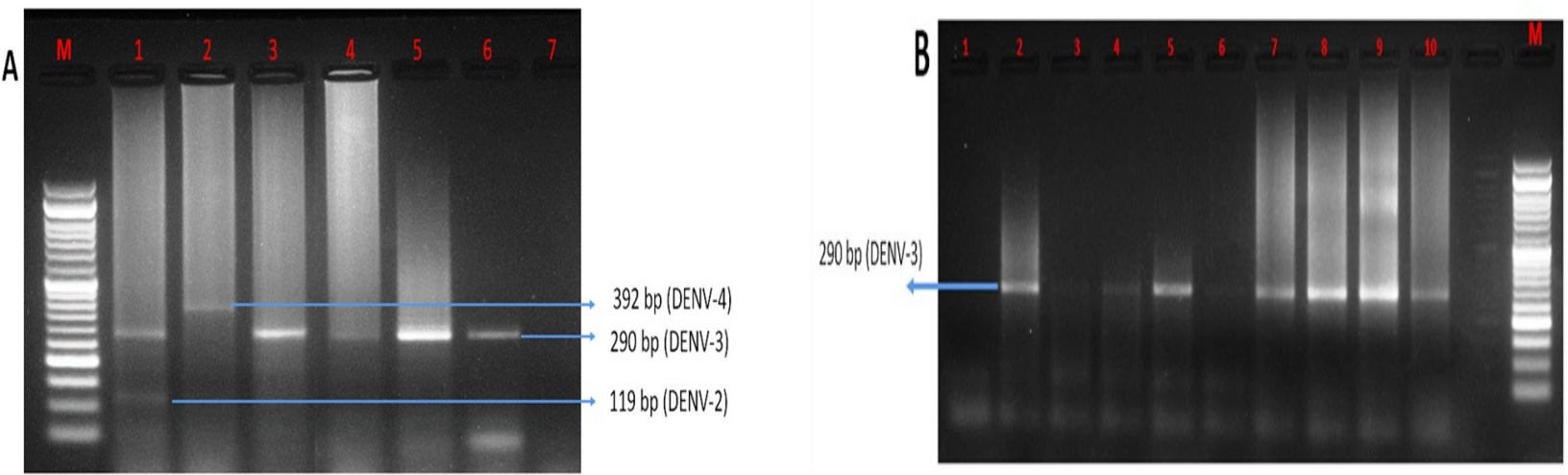

Fig. 2. Agarose gel pics (2\%) showing amplified DNA bands of DENV serotypes. (A) Lane 1-6 showed DENV 2,3, and 4 serotypes, negative control (Lane 7), and $M$ indicates a 50 bp DNA ladder. (B) DENV 3 serotype (lane 2-10) where lane 1 is a negative control.

percentage of samples found IgG positive and IgM negative was $12.73 \%(7 / 55)$ (Fig 4).

Prevalence of DENV/CHIKV co-infection during 2017-2018 in Delhi

We found the co-circulation of DENV and CHIKV in the studied patients and noticed the co-infection of both pathogens in a significant proportion. We have found the prevalence of DENV/CHIKV co-infection to $10.34 \%$ (9/87) (Table 1). Their median age was 31 years and females $(66.67 \%, n=6 / 9)$ appear to become more prone to the infection than males $(33.33 \%, n=3 / 9)$. The study of the age-wise distribution of DENV, CHIKV, and DENV/CHIKV co-infection showed that the maximum infected patients were from the age group of 18-35 years (Fig 5).

\section{DENV serotyping}

Out of 36 DENV NS1 Ag positive samples of the year 2017-2018, we were able to determine serotype for only $29(80.56 \%, 29 / 36)$ samples. The most dominant serotype during this study was DENV 3 . DENV 3 was detected in $27(75 \%, 27 / 36)$ samples whereas DENV 4 was detected in $1(2.78 \%, 1 / 36)$ sample. The infection

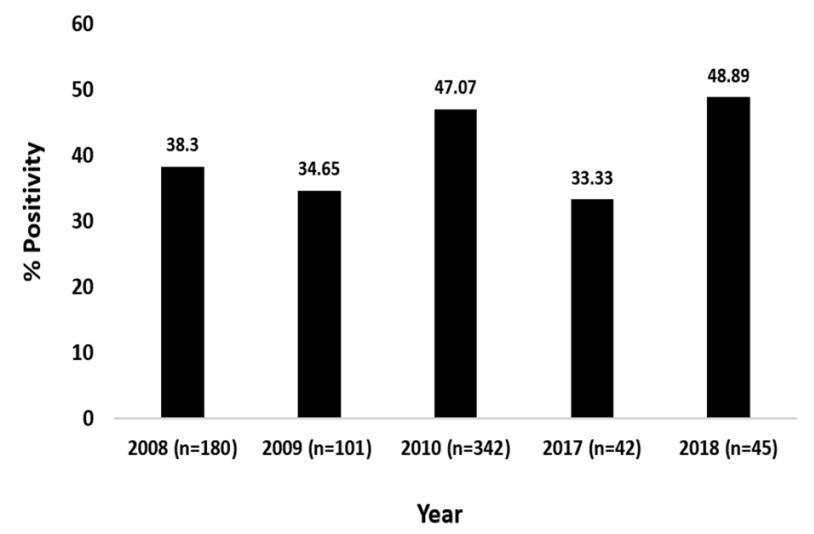

Fig. 3. Graphical representation of the year-wise prevalence of DENV infection in Delhi. of a patient with more than one serotype was observed in $1(2.78 \%, 1 / 36)$ sample only and the detected serotypes were DENV 2 and 3 . We could not find DENV 1 during this course of study. DENV 2, 3, and 4 were found circulating during 2017 whereas, during 2018 only DENV 3 was found circulating in studied samples in Delhi. Furthermore, all DENV/CHIKV co-infected patients were infected with DENV 3 serotype whereas DENV mono-infected patients were infected with DENV 3, DENV 4, and mixed serotype (DENV 2 and 3) (Fig 6). In 7 DENV NS1 Ag positive samples, infecting serotype could not be determined. On the other hand, we could determine DENV serotyping of 69 samples only collected from AlIMS during 2008-2010 and identified DENV 1 in 43.48\% (30/69) and DENV 2 in 4.35\% (3/69) samples (Fig 6). We could not find DENV 3 or DENV 4 serotype in any of the samples.

Evaluation of clinical symptoms of DENV monoinfected, CHIKV mono-infected, and DENV/CHIKV co-infected adults ( $\geq 18$ years)

Clinical symptoms were analyzed for CHIKV monoinfected, DENV mono-infected, and DENV/CHIKV coinfected (Table 2) adult patients of the year 2017-2018.

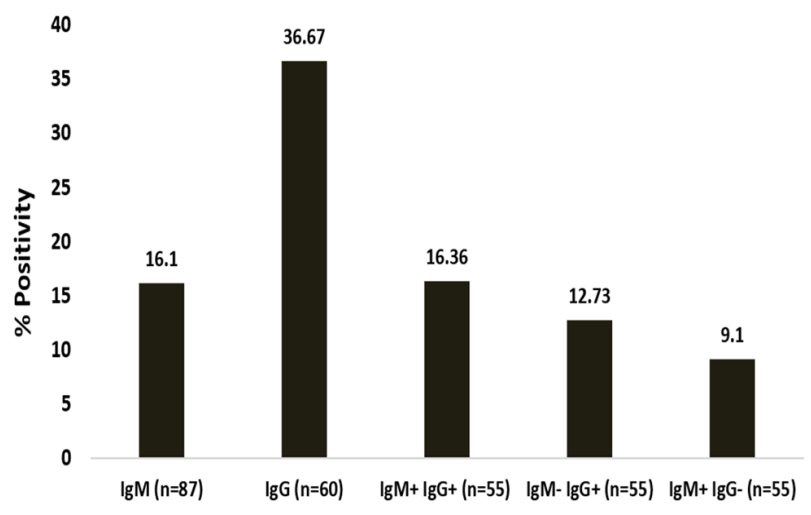

Fig. 4. Graphical representation of CHIKV patients showing the serological status of 2017-2018 collected samples. 


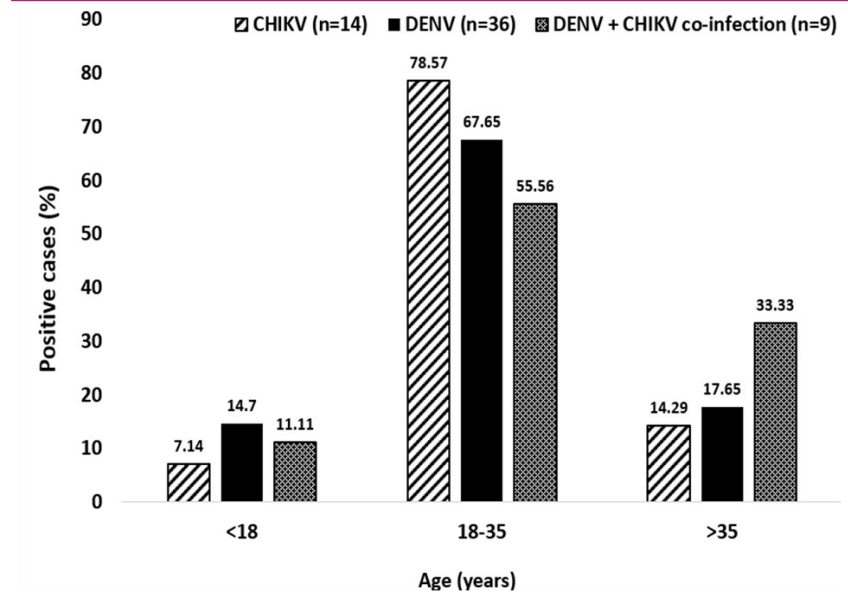

Fig. 5. Age-wise distribution of DENV, CHIKV and DENV/ CHIKV co-infection.

Although joint pain was found in all the adult groups, the relative proportion with joint pain was higher in CHIKV mono-infection compared to dengue monoinfection (75\% versus $42.86 \%$ ) but the relative proportion with joint pain was similar in CHIKV/DENV coinfection compared to CHIKV mono-infection (75\% versus $75 \%$ ). Joint swelling was found in both CHIKV mono-infection and DENV/CHIKV co-infection but was absent in DENV mono-infection. In addition to this, the relative proportion with joint swelling was double in CHIKV mono-infection as compared to CHIKV/DENV co-infection (25\% versus $12.5 \%$ ). Vomiting, however, appears to be preferential to dengue mono-infection (76.19\%) compared to either CHIKV mono-infection (50\%) or DENV/CHIKV co-infection (50\%) (Table 2).

\section{DISCUSSION}

Delhi is a dengue-endemic region and witnessed many dengue fever outbreaks. Therefore its molecular surveillance is necessary every year to make appropriate decisions can be made on existing and future strategies for proper management of dengue disease. In this study, we have found a high prevalence of dengue virus in Delhi during 2008-2010 but in 2010, the prevalence was slightly higher than 2008 and 2009, the possible reason may be the occurrence of dengue outbreak during the year and this finding is consistent with the prevalence reported from Delhi (Ahmed and Broor, 2015). In Delhi, from time to time different dengue positivity was reported as 49\% during 2011 (Afreen et al., 2014a), 69\% during 2011-2014 (Afreen et al., 2015), $71 \%$ during the 2013 outbreak (Afreen et al., 2014b), $61 \%$ during 2015 outbreak (Savargaonkar et al., 2018) and $40 \%$ during 2016 (Hisamuddin et al., 2018). But in this study during 2017 dengue cases becomes slightly lower and during 2018 again increased to $49 \%$.

DENV/CHIKV co-infection is very common in dengueendemic regions due to the overlapping epidemiology of both diseases. To know the prevalence of DENV/ CHIKV co-infection, it is necessary to know the prevalence of $\mathrm{CHIKV}$ in dengue-endemic regions. In our study, we observed a $16.1 \%$ prevalence rate of $\mathrm{CHIKV}$ which is relatively high as compared to other reports (Rezza et al., 2014; Dinkar et al., 2018; Savargaonkar et al., 2018; Murhekar et al., 2019). Due to the changing epidemiology of CHIKV, it is necessary for active

A.

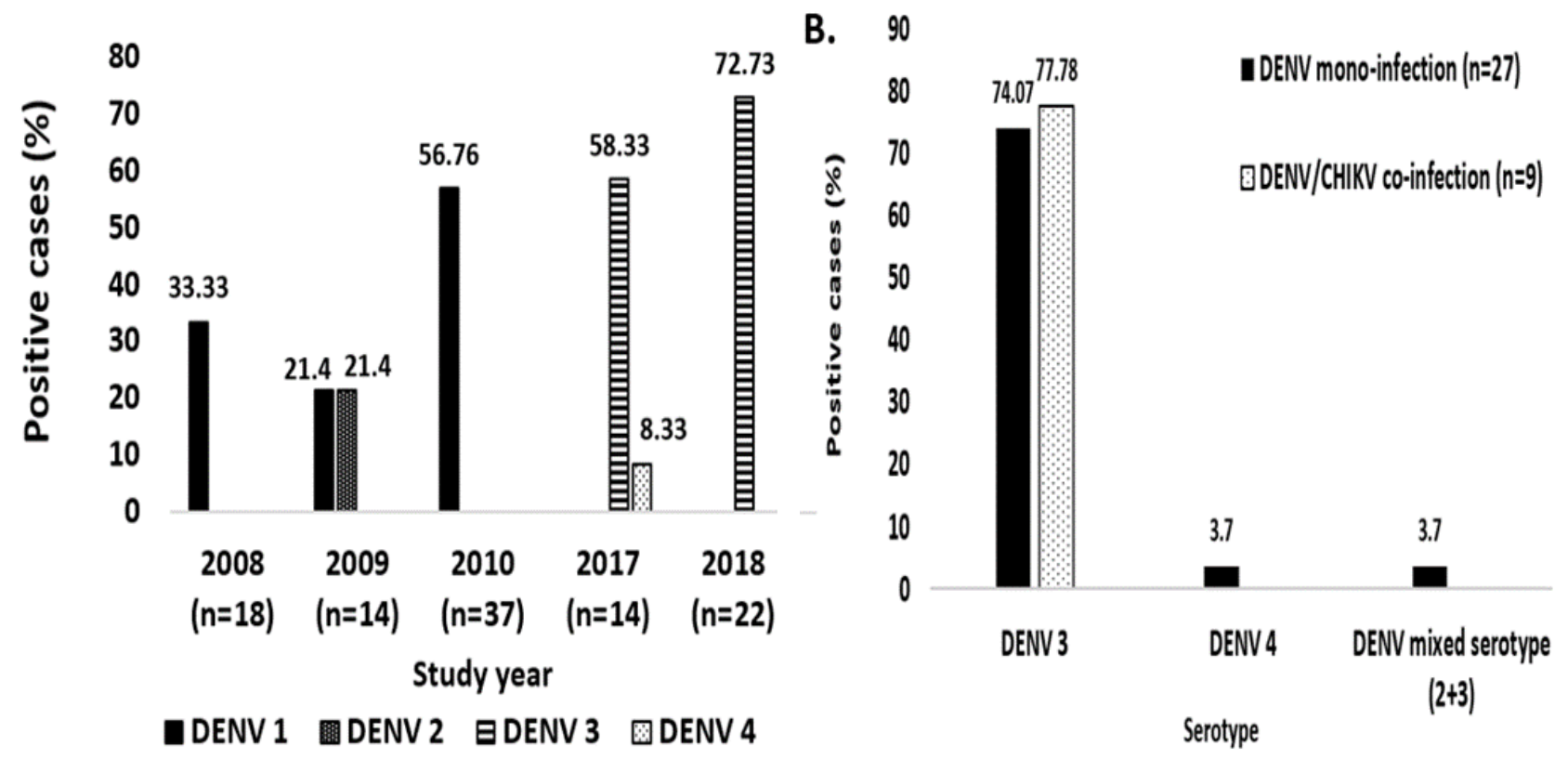

Fig. 6. A. Representation of the year-wise prevalence of DENV serotypes. B. DENV serotypes were detected in DENV mono-infection and DENV/CHIKV co-infected patients' samples collected during 2017-2018. 
molecular surveillance of CHIKV in dengue-endemic regions so that the true disease burden can be determined. To know the rough estimation of previous exposure of patients under study to CHIKV infection and also for planning CHIKV vaccine studies, prevalence data of $\lg G$ antibodies is required. As there is no robust surveillance system existing for CHIKV screening so it can be an alternative approach to screen CHIKV specific IgG towards knowing CHIKV disease burden. We have found a high prevalence of CHIKV IgG in this study which is inconsistent with a study from Pune (Patil et al., 2020). To check CHIKV's recent infection, we screened IgG positive samples for IgM positivity. Only $16.36 \%$ of samples were tested positive for the presence of IgM antibodies which indicate CHIKV infection may not have been very recently during the study. The antibody response patterns might vary between mono-infected and co-infected patients. Hence, it is very important to determine the mono-infection and coinfection status of the patients as the antibodies patterns generated in response to the infection might serve as biomarkers for disease progression (Nayak et al., 2020).

The prevalence of DENV/CHIKV co-infection was previously reported to $8.6 \%$ and $10 \%$ from Delhi during 2006 (Chahar et al., 2009) and 2011 (Afreen et al., 2014a) respectively. In our study, we have also found a high prevalence rate of DENV/CHIKV co-infection. This finding agrees with other reports from Delhi (Hisamuddin et al., 2018), Varanasi (Singh et al., 2018), Mumbai (Londhey et al., 2016), Pune (Gandhi et al., 2015). The age group of 18-35 years was observed most affected group among DENV, CHIKV, and DENV/CHIKV coinfection, and this is supported by a study from Punjab and West Bengal (Mukherjee et al., 2017; Kaur et al., 2018).

When clinical symptoms were analyzed, we observed that joint pain and joint swelling both were more in CHIKV mono-infection and DENV/CHIKV co-infection. This finding agrees with other reports (Dinkar et al., 2018; Singh et al., 2018). DENV mono-infected patients had more vomiting in comparison to CHIKV monoinfected or DENV/CHIKV co-infected patients. This is also consistent with other reports (Edwards et al., 2016; Dinkar et al., 2018; Singh et al., 2018). Thus, it appears that whenever there is a CHIKV component involved, regardless of mono or co-infection, there is a higher chance of joint pain and joint swelling. By contrast, wherever there is a CHIKV component involved regardless of mono-infection or co-infection, vomiting was relatively lower compared to dengue. These trends, although statistically insignificant, holds true even when comparing children and adult groups (data not shown). This however may be studied more deeply in future to corroborate.
A previous study on DENV/CHIKV co-infection reported that co-infection causes more severe clinical disease than mono-infection (Chahar et al., 2009; Gandhi et al., 2015), but our study did not find any clinical symptom which was associated with only DENV/CHIKV coinfection. Therefore, it is reconfirmed that DENV/CHIKV co-infection does not exacerbate the clinical severity (Caron et al., 2012; Zaidi et al., 2018; Singh et al., 2018).

Delhi witnessed many dengue fever episodes every year which were attributed to a particular dengue virus serotype. During this study, we have identified DENV 3 as a dominant serotype which is consistent with other reports from Delhi during 2016 (Parveen et al., 2019). In Delhi during 2006, DENV 2 was a dominant serotype. It was replaced by DENV 1 during 2007-2009 (Chakravarti et al., 2010) and became dominant in the 2010 outbreak (Ahmed and Broor, 2015). Then DENV 2 became dominant from 2011-2015 (Afreen et al., 2014a, Afreen et al., 2014b., Afreen et al., 2015). To the best of our knowledge, no dengue fever epidemics are attributed to DENV 4 in Delhi. DENV 2 was the predominant serotype in monotypic DENV patients in West Bengal during 2014-2015 (Mukherjee et al., 2017; Savargaonkar et al., 2018). To know whether any particular serotype is associated with DENV/CHIKV coinfection, we determined the serotyping of co-infected samples also. We found DENV 3 serotype in all DENV/ CHIKV co-infected patients. In contrary to this, reports from Delhi and Laos evidenced the different DENV serotypes in co-infected patients (Afreen et al., 2014a; Phommanivong et al., 2016). A report from Delhi evidenced circulation of DENV 2 followed by DENV 3 as the major serotype in DENV/CHIKV co-infected patients during 2011 (Afreen et al., 2014a). Previously, it has been reported that DENV 3 infected patients showed hepatomegaly (Kumaria, 2010) and elevated levels of liver enzymes, AST, and/or ALT in the DHF condition (Rezza et al., 2014). Infection of patients with multiple DENV serotypes was reported more likely to have clinical severity as compared to patients infected with only one serotype (Dhanoa et al., 2016). The change in serotyping pattern and clinical severity with multiple serotypes necessitates the determination of DENV serotyping over the period which is necessary for proper preventive measures and clinical management of patients and vaccine trials. Although we have reported only one case of concurrent infection with multiple serotypes in Delhi due to hyper-endemicity, the cocirculation of multiple serotypes in Delhi is very common (Afreen et al., 2014b). Although reasons remain to be fully understood, it is presumed that due to low viral titer, we could not determine the serotype of 7 DENV NS1 Ag positive samples. 


\section{Conclusion}

Our prevalence data highlights the need for active surveillance of DENV/CHIKV co-infection especially in the endemic regions for both viruses. Our study also highlights that DENV/CHIKV co-infection does not always involve the exacerbation of clinical features. Our study provides the prevalence data on CHIKV and DENV and dengue virus serotyping which is required for understanding the epidemiology of both arboviral diseases and will be helpful for vaccine developers and proper clinical management of CHIKV and DENV infected patients. Epidemiological data of any disease plays a significant role in making better policies and initiations of any vaccine trials by the health workers. Therefore, epidemiological surveillance has a key role in proper disease management. This study found the prevalence of DENV (41.38\%), CHIKV (16.1\%), and DENV/CHIKV coinfection $(10.34 \%)$. Sometimes, secondary infection with DENV heterologous serotypes\{(Dengue Haemorrhagic Fever (DHF) and Dengue Shock Syndrome (DSS)\} may lead to complications and even death of the infected patients. So, tracking the DENV serotype is very important for the proper management of DENV patients. The Dominance of DENV serotypes $(1,2,3)$ varied in different time periods of our study. Clinical symptoms revealed that DENV/CHIKV co-infection did not exacerbate the clinical severity compared to DENV and CHIKV mono-infected patients.

\section{Declarations \\ Funding}

This study partially received financial support from the Department of Biotechnology, Government of India during 2008-2010 but we did not receive any specific grant from funding agencies in the public, commercial, or not-for-profit sectors during the study period 2017 2018.

\section{Compliance with ethical standards}

This study was approved by the Institutional Ethical Committee of Jamia Hamdard, New Delhi, and AlIMS, New Delhi. The procedure used in this study adheres to the tenets of the declaration of Helsinki. Human patient's blood samples were collected from all patients by taking written informed consent.

\section{Consent to participate}

Informed written consent was taken from all patients who participated in the study.

\section{Author's contributions}

RS performed the experiments and analyzed the data and wrote the initial manuscript. VJ helped in sample collection. AC, MK, and AKP helped in the clinical study design. SR and $\mathrm{KN}$ reviewed the initial manuscript. KKG reviewed the manuscript, helped in data analysis, editing and finalizing the manuscript. NK and PR supervised the whole study including study design and final editing of the manuscript. All authors read and approved the final manuscript.

\section{ACKNOWLEDGEMENTS}

RS acknowledges the JRF fellowship from the University Grant Commission, Govt. of India. The authors gratefully acknowledge Ms. Shipra Gupta for useful comments on the manuscript.

\section{Conflict of interest}

The authors declare that they have no conflict of interest.

\section{REFERENCES}

1. Afreen, N., Deeba, F., Khan, W. H., Haider, H. S., Kazim, S. N., Ishrat, R, Naqvi I. H., Shareef, M. Y., Broor, S., Ahmed, A. \& Parveen, S. (2014a). Molecular characterization of dengue and chikungunya virus strains circulating in New Delhi, India. Microbiol Immunol., 58:688-696. https:// doi.org/10.1111/1348-0421.12209

2. Afreen, N., Deeba, F., Naqvi, I., Shareef, M., Ahmed, A., Broor, S., \& Parveen, S. (2014b). Molecular investigation of 2013 dengue Fever outbreak from delhi, India. PLoS Curr 6:. https://doi.org/10.1371/currents.outb reaks.0411 252a8b82aa933f6540abb54a855f

3. Afreen, N., Naqvi, I. H., Broor, S., Ahmed, A. \& Parveen, S. (2015). Phylogenetic and Molecular Clock Analysis of Dengue Serotype 1 and 3 from New Delhi, India. PLOS ONE, 10:e0141628. https://doi.org/10.1371/journal.po ne. 0141628

4. Ahmed, N. \& Broor, S. (2015). Dengue Fever Outbreak in Delhi, North India: A Clinico-Epidemiological Study. Indian journal of community medicine: official publication of Indian Association of Preventive \& Social Medicine, 40,135138. https://doi.org/10.4103/0970-0218.153884

5. Azeredo, E. L., Dos Santos, F. B., Barbosa, L. S., Alva Souza, T.M., Badolato-Correa, J., Sanchez-Arcila, J.C., Nunes, P.C.G., De-Oliveira-Pinto, L.M., De Filippis, A.M., Fabbro, M.D., Romanholi, I.H., \& Da Cunha, R.V. (2018). Clinical and Laboratory Profile of Zika and Dengue Infected Patients: Lessons Learned From the Co-circulation of Dengue, Zika and Chikungunya in Brazil. PLoS Curr., 10. https://doi.org/10.1371/ currents.outbreaks.0bf6aeb4d30824de63c4d5d745b217f5

6. Burt, F. J., Rolph, M. S., Rulli, N. E., Mahalingam, S. \& Heise, M. T. (2012). Chikungunya: a re-emerging virus. Lancet, 379,662-671. https://doi.org/10.1016/S0140-6736 (11)60281-X

7. Caron, M., Paupy, C., Grard, G., Becquart,P., Mombo, I., Nso, B. B. B., Kassa, F. K., Nkoghe, D. \& Leroy, E. M. (2012). Recent introduction and rapid dissemination of Chikungunya virus and Dengue virus serotype 2 associated with human and mosquito coinfections in Gabon, central Africa. Clin. Infect. Dis., 55,e45-53. https://doi.org/1 0.1093/cid/cis530 
8. Chahar, H. S., Bharaj, P., Dar, L., Guleria, R., Kabra, S. K. \& Broor, S. (2009). Co-infections with chikungunya virus and dengue virus in Delhi, India. Emerging Infect Dis., 15,1077-1080. https://doi.org/10.3201/eid1507.080638

9. Chakravarti, A., Kumar, A. \& Matlani, M. (2010). Displacement of dengue virus type 3 and type 2 by dengue virus type 1 in Delhi during 2008. Indian J Med Microbiol 28:412. https://doi.org/10.4103/0255-0857.71806

10. Dhanoa, A., Hassan, S. S., Ngim. C.F., Lau, C. F., Chan,T.S., Adnan, N. A. A., Eng, W. W. H., Gan, H. M. \& Rajasekaram, G. (2016). Impact of dengue virus (DENV) co-infection on clinical manifestations, disease severity and laboratory parameters. BMC Infect Dis., 16,.. https:// doi.org/10.1186/s12879-016-1731-8

11. Dinkar, A., Singh, J., Prakash, P., Das, A. \& Nath, G. (2018). Hidden burden of chikungunya in North India; A prospective study in a tertiary care centre. $J$ Infect Public Health, 11,586-591. https://doi.org/10.1016/j.jiph.2017.0 9.008

12. Edwards, T., Signor, L. D. C. C., Williams C., Donis, E., Cuevas, E. L. \& Adams, E. R. (2016). Co-infections with Chikungunya and Dengue Viruses, Guatemala, 2015. Emerging Infect Dis., 22,2003-2005. https://doi.o $\mathrm{rg} / 10.3201 / \mathrm{eid} 2211.161017$

13. Furuya-Kanamori, L., Liang, S., Milinovich, G., Magalhaes, R. J. S., Clements, A.C.A., Hu, W., Brasil, P., Frentiu, F.D., Dunning, R. \& Yakob, L. (2016). Codistribution \& co-infection of chikungunya and dengue viruses. BMC Infect Dis., 16,84. https://doi.org/10.1186/ s12879-016-1417-2

14. Gandhi, B. S., Kulkarni, K., Godbole, M. \& Dole, S.S. (2015). Dengue and chikungunya co-infection associated with more severe clinical disease than mono-infection. International Journal of Healthcare and Biomedical Research, 3:117-23

15. Gubler, D.J. (1998). Dengue and Dengue Hemorrhagic Fever. Clin Microbiol Rev 11:480-496

16. Hammon, W. M., Rundnick, A. \& Sather, G. E. (1960). Viruses Associated with Epidemic Hemorrhagic Fevers of the Philippines and Thailand. Science 131:1102-1103. https://doi.org/10.1126/science.131.3407.1102

17. Hasebe, F., Parquet, M. C., Pandey, B. D., Mathenge, E. G. M., Morita, K., Balasubramaniam, V., Saat, Z., Yusop, A., Sinniah, M., Natkunam, S. \& Igarashi, A. (2002). Combined detection and genotyping of Chikungunya virus by a specific reverse transcription-polymerase chain reaction. Journal of Medical Virology, 67, 370-374. https:// doi.org/10.1002/jmv.10085

18. Hisamuddin, M., Tazeen, A., Abdullah, M., Islamuddin, M., Parveen, N., Islam, A., Faizan, M. I., Hamza, A., Naqvi, I. H., Verma, H. N., Malik, A., Ahmed, A. \& Parveen, S. (2018). Co-circulation of Chikungunya and Dengue viruses in Dengue endemic region of New Delhi, India during 2016. Epidemiol Infect., 146, 1642-1653. https://doi.org/1 $0.1017 /$ S0950268818001590

19. Kaur, M., Singh, K., Sidhu, S.K., Devi, P., Kaur, M., Soneja, S. \& Singh, N. (2018). Coinfection of chikungunya and dengue viruses: A serological study from North Western region of Punjab, India. J Lab Physicians, 10,443447. https://doi.org/10.4103/JLP.JLP_13_18

20. Kumari, R., Kumar, K. \& Chauhan, L. S. (2011). First dengue virus detection in Aedes albopictus from Delhi, India: its breeding ecology and role in dengue transmission. Trop. Med. Int. Health, 16, 949-954. https://doi.org/1 0.1111/j.1365-3156.2011.02789.x

21. Kumaria, R. (2010). Correlation of disease spectrum among four Dengue serotypes: a five years hospital based study from India. Braz J Infect Dis 14:141-146

22. Lanciotti, R.S., Calisher, C. H., Gubler, D. J., Chang, G.J. \& Vorndam, A. V. (1992). Rapid detection and typing of dengue viruses from clinical samples by using reverse transcriptase-polymerase chain reaction. J. Clin. Microbiol., 30, 545-551

23. Londhey, V., Agrawal, S., Vaidya, N., Kini, S., Shastri, J. S. \& Sunil, S. (2016). Dengue and Chikungunya Virus Coinfections: The Inside Story. J Assoc Physicians India 64:36-40

24. Medina, F., Medina, J. F., Colón, C., Vergne, E., Santiago, G,A., \& Munoz-Jordan, J.L. (2012). Dengue Virus: Isolation, Propagation, Quantification, and Storage. Current protocols in microbiology Chapter 15:Unit15D.2. https:// doi.org/10.1002/9780471729259.mc15d02s27

25. Mukherjee, S,, Dutta, S, K., Sengupta, S., Tripathi, A. (2017). Evidence of dengue and chikungunya virus coinfection and circulation of multiple dengue serotypes in a recent Indian outbreak. Eur. J. Clin. Microbiol. Infect. Dis., 36, 2273-2279. https://doi.org/10.1007/s10096-017-3061-1

26. Murhekar, M., Kanagasabai, K., Shete, V., Joshua, V., Ravi, M., Kirubakran, B. K., Ramachandran, R., Sabarinathan. \& Gupta, N. (2019). Epidemiology of chikungunya based on laboratory surveillance data-India, 2016-2018. Trans. R. Soc. Trop. Med. Hyg., 113,259-262. https:// doi.org/10.1093/trstmh/try141

27. Nayak, K., Jain, V., Kaur, M., Khan, N., Gottimukkala, K., Aggarwal,C., Sagar, R., Gupta, S., Rai, R. R., Dixit, K., Islamuddin, M., Khan, W. H., Verma, A., Maheshwari, D., Chawla, Y. M., Reddy, E. S., Panda, H., Sharma, P., Bhatnagar, P., Singh, P., Raghavendhar B, S., Patel, A. K., Ratageri, V. H., Chandele, A., Ray, P. \& MuraliKrishna, K. (2020). Antibody response patterns in chikungunya febrile phase predicts protection versus progression to chronic arthritis. $\mathrm{JCl}$ Insight. https:// doi.org/10.1172/jci.insight.130509

28. National Vector Borne Disease Control Programme. In: Dengue Cases and Deaths in the Country since 2015. https://nvbdcp.gov.in/index4.php?

lang $=1 \&$ level $=0 \&$ linkid $=431 \&$ lid $=3715$. Accessed 29 Aug 2020

29. National Vector Borne Disease Control programme. In: Epidemiological Profile of Chikungunya Fever in the Country since 2015. https://nvbdcp.gov.in/index4.php? lang=1\&level=0\&linkid=486\&lid=3765. Accessed 29 Aug 2020

30. Palomares-Reyes, C., Silva-Caso, W., Del Valle, L. J., Aguilar-Luis, M. A., Weilg, C., Martins-Luna, J., VinasOspino, A., Stimmler, L., Espinoza, N. M., Ortega, R.A., Espiritu, W. E., Misaico, E. \& De Valle-Mendoza, J. (2019). Dengue diagnosis in an endemic area of Peru: Clinical characteristics and positive frequencies by RTPCR and serology for NS1, IgM, and IgG. Int. J. Infect Dis., 81,31-37. https://doi.org/10.1016/j.ijid.2019.01.022

31. Parveen, N., Islam, A., Tazeen, A., Hisamuddin, M., Abdullah, M., Naqvi, I. H., Faizan, M. I., Gulyani, D., Ahmed, A. \& Parveen, S. (2019). Circulation of single serotype of 
Dengue Virus (DENV-3) in New Delhi, India during 2016: A change in the epidemiological trend. J. Infect Public Health, 12, 49-56. https://doi.org/10.1016/j.jiph.2018.0 8.008

32. Patil, H. P., Rane, P. S., Gosavi, M., Mishra, A. C. \& Arankalle, V. A. (2020). Standardization of ELISA for antichikungunya-IgG antibodies and age-stratified prevalence of anti-chikungunya-IgG antibodies in Pune, India. Eur. J. Clin. Microbiol. Infect Dis., https://doi.org/10.1007/s10096020-03933-5

33. Phommanivong, V., Kanda, S., Shimono, T., Laaningao, P., Darcy, A.W., Mishima, N., Phaytanavanh, B \& Nishiyama, T. (2016). Co-circulation of the dengue with chikungunya virus during the 2013 outbreak in the southern part of Lao PDR. Tropical Medicine and Health, 44, 24. https:// doi.org/10.1186/s41182-016-0020-y

34. Ray, P., Ratagiri, V. H., Kabra, S. K., Lodha, R., Sharma S., Sharma, B. S., Kalaivani, M., Wig, N. (2012). Chikungunya infection in India: results of a prospective hospital based multi-centric study. PLOS ONE, 7,e30025. https://doi.org/10.1371/journal.pone.0030025

35. Requena-Castro, R., Reyes-López, M. Á., RodríguezReyna, R. E., Pailma-Nicolas, P. \& Bocanegra-Garcia, V. (2017). Molecular detection of mixed infections with multiple dengue virus serotypes in suspected dengue samples in Tamaulipas, Mexico. Mem Inst Oswaldo Cruz., 112,520 -522. https://doi.org/10.1590/0074-02760160468

36. Rezza, G., El-Sawaf, G., Faggioni, G., Vescio, F., Santis, R. D., Helaly, G., Pomponi, A., Metwally, D., Fantini, M., Qudi, H., Ciccozzi, M. \& Lista, F. (2014). Co-circulation of Dengue and Chikungunya Viruses, Al Hudaydah, Yemen, 2012. Emerg Infect Dis., 20,1351-1354. https:// doi.org/10.3201/eid2008.131615

37. Rosso, F., Pacheco, R., Rodríguez, S. \& Bautista, D. (2016). Co-infection by Chikungunya virus (CHIK-V) and dengue virus (DEN-V) during a recent outbreak in Cali, Colombia: Report of a fatal case. Rev Chilena Infectol., 33,464-467.https://doi.org/10.4067/S0716-101820160 00400013

38. Savargaonkar, D., Sinha, S., Srivastava, B., Nagpal, B.
N., Sinha, A., Shamim, A., Das, R., Pande, V., Anvikar, A. R. \& Valecha, N. (2018). An epidemiological study of dengue and its coinfections in Delhi. International Journal of Infectious Diseases, 74, 41-46. https://doi.org/10.1016/ j.ijid.2018.06.020

39. Simon, F., Parola, P., Grandadam, M., Babrina, F., Manuela, O., Philippe, B., Pierre, H., Philippe, K., Ali, A. A., Xavier, D.L., Remi, C., \& Hugues, T. (2007). Chikungunya infection: an emerging rheumatism among travelers returned from Indian Ocean islands. Report of 47 cases. Medicine (Baltimore) 86,123-137. https://doi.org/10.1097/ MD/0b013e31806010a5

40. Singh, J., Dinkar, A., Singh, R. G., Siddiqui, M. S., Sinha, N. \& Singh, S. K. (2018). Clinical profile of dengue fever and coinfection with chikungunya. Ci Ji Yi Xue Za Zhi 30,158-164. https://doi.org/10.4103/tcmj.tcmj_138_17

41. Singh, P., Mittal, V., Rizvi, M. M. A., Chhabra, M., Sharma, P., Rawat, D. S., Bhattacharya, D., Chauhan, L. S. \& Rai, A. (2012). The first dominant co-circulation of both dengue and chikungunya viruses during the postmonsoon period of 2010 in Delhi, India. Epidemiol. Infect, 140,1337-1342.https://doi.org/10.1017/S0950268811 001671

42. Subudhi, B. B., Chattopadhyay, S., Mishra, P., \& Kumar, A. (2018). Current Strategies for Inhibition of Chikungunya Infection. Viruses 10:. https://doi.or g/10.3390/v10050235

43. Taraphdar, D., Sarkar, A., Mukhopadhyay, B. B. \& Chatterjee, S. (2012). A Comparative Study of Clinical Features between Monotypic and Dual Infection Cases with Chikungunya Virus and Dengue Virus in West Bengal, India. The American Journal of Tropical Medicine and Hygiene, 86,720-723. https://doi.org/10.4269/ajtmh.201 $2.11-0704$

44. Zaidi, M. B., Garcia-Cordero, J., Rivero-Gomez, R., Corzo -Gomez, J., Almeida, M. E. G. Y., Bonilla-Moreno, R., Bustos-Arriaga, J., Villegas-Sepulveda, N., Flores-Romo, L \& Cedillo-Barron, L. (2018). Competitive suppression of dengue virus replication occurs in chikungunya and dengue co-infected Mexican infants. Parasit Vectors 11:378. https://doi.org/10.1186/s13071-018-2942-1 